\title{
Flavour physics at CLEO-c
}

\author{
J. Libbya (on behalf of the CLEO collaboration)

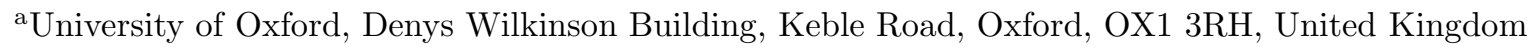

Three CLEO-c results related to flavour-physics are presented: the determination of the strong-phase difference between $D^{0} \rightarrow K^{+} \pi^{-}$and $D^{0} \rightarrow K^{-} \pi^{+}, \delta$, the measurement of the coherence parameter and average strongphase difference between $D^{0} \rightarrow K^{+} \pi^{-} \pi^{-} \pi^{+}$and $D^{0} \rightarrow K^{-} \pi^{+} \pi^{+} \pi^{-}$and measurements of the variation of strongphase difference between $D^{0}$ and $\overline{D^{0}}$ decays to $K_{S}^{0} \pi^{+} \pi^{-}$over phase space. All measurements are important for determining the unitarity triangle angle $\gamma$ from $B \rightarrow D^{(*)} K^{(*)}$ decays. Furthermore, the measurement of $\delta$ is important for interpreting $D^{0}-\overline{D^{0}}$ mixing.

\section{Introduction}

The precise determination of the unitarity triangle angle $\gamma$ is a principal goal of flavour physics. In particular, measurements of $\gamma$ from tree-level processes, which are insensitive to Beyond the Standard Model (BSM) physics, can be compared to measurements in loop processes, which are sensitive to virtual corrections from BSM physics. The current average of tree-level determinations of $\gamma$ is $\left(77_{-32}^{+30}\right)^{\circ}[1]$. The most precise determinations to date come from measurements of direct $C P$ violation in $B^{ \pm} \rightarrow D K^{ \pm}$, where the $D$ is a $D^{0}$ or $\overline{D^{0}}$ decaying to the same final state. Determinations of $\gamma$ from such measurements require knowledge of the strong decay parameters of the $D^{0}$ and $\overline{D^{0}}$, in particular the strong-phase differences.

Strong-phase differences and other important parameters of multi-body $D$ decay, relevant to $\gamma$ measurements, can be studied in quantumcorrelated $D^{0} \overline{D^{0}}$ pairs produced in $e^{+} e^{-}$collisions at a centre-of-mass energy equal to the $\psi(3770)$ mass. The charge-conjugation quantum number $(C)$ of the initial and final states is -1 . Therefore, if one $D$ decays into a $C P$ eigenstate such as $\pi^{+} \pi^{-}$this identifies the other $D$ to be in a state of opposite $C P$. The $C P$-tagged rates of decays are sensitive to the strong-phase differences required for $\gamma$ measurements. Furthermore, using multi-body $D$ decays to determine $\gamma$ requires knowledge of additional parameters related to the presence of intermediate resonances; these can also be determined from quantum-correlated $D$-decay data.

Studies of $D \rightarrow K^{-} \pi^{+}$decays at the $\psi(3770)$ are also sensitive to the $D^{0}-\bar{D}^{0}$ mixing parameters as well as the strong-phase difference between doubly Cabbibo suppressed (DCS) and Cabbibo favoured $(\mathrm{CF})$ decays $\delta$, which is defined by the relation $\left\langle K^{+} \pi \mid D^{0}\right\rangle /\left\langle K^{-} \pi^{+} \mid D^{0}\right\rangle=r e^{i \delta}$, where $r=0.0616$ [2] is the absolute value of the amplitude ratio.

These proceedings describe three measurements by CLEO-c of quantum-correlated $D$ decay. Section 2 describes the CLEO-c experiment and data sets used for the analyses. Section 3 presents a measurement of charm mixing parameters and $\delta$. Sections 4 and 5 describe measurements of strong parameters related to $\gamma$ measurements in the multi-body decays $D \rightarrow$ $K^{ \pm} \pi^{\mp} \pi^{+} \pi^{-}$and $D \rightarrow K_{S}^{0} \pi^{+} \pi^{-}$, respectively.

\section{CLEO-c}

All measurements presented are made with $e^{+} e^{-} \rightarrow \psi(3770)$ data accumulated at the Cornell Electron Storage Ring (CESR). The CLEO-c detector is used to collect these data, which is described in detail elsewhere $\underline{3}$. The total integrated luminosity of the data is $818 \mathrm{pb}^{-1}$, however, only $218 \mathrm{pb}^{-1}$ have been used so far for the measurement of $\delta$ presented in Section 3 . 
Table 1

Correlated (C-odd) effective $D^{0} \bar{D}^{0}$ branching fractions to leading order in conjugate modes are applied. The rates are normalised to the multiple of the uncorrelated branching fractions.

\begin{tabular}{lc}
\hline Mode & Correlated branching fraction \\
\hline$K^{-} \pi^{+}$vs. $K^{-} \pi^{+}$ & $R_{M}$ \\
$K^{-} \pi^{+}$vs. $K^{+} \pi^{-}$ & $\left(1+R_{W S}\right)^{2}-4 r \cos \delta(r \cos \delta+y)$ \\
$K^{-} \pi^{+}$vs. $S_{ \pm}$ & $1+R_{W S} \pm 2 r \cos \delta \pm y$ \\
$K^{-} \pi^{+}$vs. $e^{-}$ & $1-r y \cos \delta-r x \sin \delta$ \\
$S_{ \pm}$vs. $S_{ \pm}$ & 0 \\
$S_{+}$vs. $S_{-}$ & 4 \\
$S_{ \pm}$vs. $e^{-}$ & $1 \pm y$ \\
\hline
\end{tabular}

\section{Measurement of mixing and the strong- phase difference in $D \rightarrow K^{-} \pi^{+}$}

Charm mixing is described by two dimensionless parameters $x \equiv\left(M_{2}-M_{1}\right) / \Gamma$ and $y \equiv$ $\left(\Gamma_{2}-\Gamma_{1}\right) / 2 \Gamma$, where $M_{1,2}$ and $\Gamma_{1,2}$ are the masses and widths, respectively, of the $C P-\left(D_{1}\right)$ and $C P+\left(D_{2}\right)$ neutral $D$ meson eigenstates and $\Gamma \equiv$ $\left(\Gamma_{1}+\Gamma_{2}\right) / 2$. Mixing has been probed in lifetime measurements to $C P$-eigenstates and in DCS decays 11 However, when measuring mixing with the DCS decay $D^{0} \rightarrow K^{+} \pi^{-}$the quantities measured are $R_{M} \equiv\left(x^{2}+y^{2}\right) / 2$ and $y^{\prime} \equiv y \cos \delta-x \sin \delta$. Therefore, without a direct determination of $\delta$ it is impossible to combine or compare the determinations of $y$ from other measurements to those from $D^{0} \rightarrow K^{+} \pi^{-}$. Furthermore, constraining $\delta$ is an important input to measuring $\gamma$ from $B^{ \pm} \rightarrow D\left(K^{+} \pi^{-}\right) K^{ \pm}$decays 5 ; this method is discussed further in Section 4

The first measurements of the parameters $y$ and $\cos \delta$ in quantum-correlated $\psi(3770)$ data are presented; the method, which is known as The Quantum Correlated Analysis (TQCA), follows that described in Ref. [6]. The parameters are extracted by comparing the decay rates where either one (single-tagged) or both (double-tagged) neutral $D$ decays are reconstructed. The effective double-tagged rates for different final states are summarised in Table 1 where $S^{ \pm}$and $e^{ \pm}$are used to indicate $C P \pm$ eigenstates and semileptonic final states, respectively; some rates depend on the $D^{0} \rightarrow K^{-} \pi^{+}$wrong-sign rate ra-

\footnotetext{
${ }^{1}$ For a review of charm mixing results see Ref. [4].
}

tio, $R_{W S} \equiv r^{2}+r y^{\prime}+R_{M}$. The most striking consequences of the quantum correlations are the enhancements and suppression of the $D^{0} \overline{D^{0}}$ decaying to opposite and the same $C P$ eigenstates, respectively.

Table 2

Final states reconstructed in TQCA.

\begin{tabular}{lc}
\hline Type & Final states \\
\hline Flavoured & $K^{-} \pi^{+}, K^{+} \pi^{-}$ \\
$S^{+}$ & $K^{+} K^{-}, \pi^{+} \pi^{-}, K_{S}^{0} \pi^{0} \pi^{0}, K_{L}^{0} \pi^{0}$ \\
$S^{-}$ & $K_{S}^{0} \pi^{0}, K_{S}^{0} \eta, K_{S}^{0} \omega$ \\
$e^{ \pm}$ & Inclusive $X e^{+} \nu, X e^{-}$ \\
\hline
\end{tabular}

The analysis has been performed with $218 \mathrm{pb}^{-1}$ of $\psi(3770)$ data 7 . The single and double-tag rates have been determined for the final states listed in Table 2. Hadronic final states without a $K_{L}^{0}$ are fully reconstructed via two kinematic variables: the beam-constrained candidate mass $M \equiv \sqrt{E_{\text {beam }}^{2}-\mathbf{p}_{D}^{2}}$ and $\Delta E \equiv E_{D}-E_{\text {beam }}$, where $E_{\text {beam }}$ is the beam energy, $\mathbf{p}_{D}$ and $E_{D}$ are the $D^{0}$ candidate momentum and energy, respectively. The yields are extracted from the one or two dimensional $M$ distributions for single and double-tagged events, respectively. The reconstruction of $K_{L}^{0} \pi^{0}$ events utilises the missing-mass technique described in Ref. [8]. The inclusive $e^{ \pm}$ tags exploit electrons identified using a multivariate discriminant $[9$.

The standard fit to determine the mixing parameters and $\cos \delta$ includes independent, external measurements of $R_{M}, R_{W S}$ and uncorrelated branching fractions. Additional external measurements of charm mixing are included in an extended fit which allows $x \sin \delta$ to also be determined. The fit accounts for correlations amongst the inputs and any cross-feed between the signal channels.

The results of the standard and extended fit are given in Table 3. A value of $x \sin \delta$ can only be determined reliably in the extended fit. The extended fit likelihoods for $\cos \delta, x \sin \delta$ and $\delta$ are shown in Figure 1. Despite the additional information in the extended fit the uncertainty on $\cos \delta$ increases due to the non-linear relationship between $\cos \delta$ and $y$. However, the determination of 
Table 3

TQCA results from the standard and extended fit. Uncertainties are statistical and systematic, respectively.

\begin{tabular}{lcc}
\hline Parameter & Standard fit & Extended fit \\
\hline$y\left(10^{-3}\right)$ & $-45 \pm 59 \pm 15$ & $6.5 \pm 0.2 \pm 0.1$ \\
$r^{2}\left(10^{-3}\right)$ & $8.0 \pm 6.8 \pm 1.9$ & $3.44 \pm 0.01 \pm 0.09$ \\
$\cos \delta$ & $1.03 \pm 0.19 \pm 0.06$ & $1.10 \pm 0.35 \pm 0.07$ \\
$x^{2}\left(10^{-3}\right)$ & $-1.5 \pm 3.6 \pm 4.2$ & $0.06 \pm 0.01 \pm 0.05$ \\
$x \sin \delta\left(10^{-3}\right)$ & 0 (fixed) & $4.4 \pm 2.4 \pm 2.9$ \\
\hline
\end{tabular}

$x \sin \delta$ in the extended fit allows the likelihood for $\delta$ to be determined. This leads to a measurement of $\delta=\left(22_{-12-11}^{+11+9}\right)^{\circ}$, which is the first direct determination. These measurements are important for the interpretation of the combined results on charm mixing [10].

\section{Measurement of the coherence parame- ter and average strong-phase difference in $D \rightarrow K \pi \pi \pi$}

The amplitudes for $B^{-} \rightarrow D^{0} K^{-}$and $B^{-} \rightarrow$ $\overline{D^{0}} K^{-}$have a relative phase between them of $\delta_{B}-\gamma$, where $\delta_{B}$ is the strong-phase difference. For decays in which the $D^{0}$ and $\overline{D^{0}}$ decay to the same final state, $f_{D}$, the two amplitudes interfere giving sensitive to $\gamma$. The amplitude for $B^{-} \rightarrow D\left(f_{D}\right) K^{-}$is given by:

$A\left(B^{-} \rightarrow D\left(f_{D}\right) K^{-}\right) \propto A_{D^{0}}+r_{B} e^{i\left(\delta_{B}-\gamma\right)} A_{\overline{D^{0}}},(1)$

where $r_{B}<0.13$ at $90 \%$ c.l. [1] is the ratio of the absolute value of $B^{-} \rightarrow \overline{D^{0}} K^{-}$to $B^{-} \rightarrow D^{0} K^{-}$ amplitudes, and $A_{D^{0}}$ and $A_{\overline{D^{0}}}$ are the amplitudes for $D^{0} \rightarrow f_{D}$ and $\overline{D^{0}} \rightarrow f_{D}$, respectively.

For the case where $f_{D}$ is $K^{-} \pi^{+}[5]$ the decay rates are equal to:

$$
\begin{aligned}
\Gamma_{B^{-} \rightarrow D\left(K^{-} \pi^{+}\right) K^{-}} & \propto 1+\left(r_{B} r\right)^{2} \\
& +2 r_{B} r \cos \left(\delta_{B}-\delta-\gamma\right), \\
\Gamma_{B^{-} \rightarrow D\left(K^{+} \pi^{-}\right) K^{-}} & \propto r_{B}^{2}+r^{2} \\
& +2 r_{B} r \cos \left(\delta_{B}+\delta-\gamma\right) .
\end{aligned}
$$

The suppressed rate has an interference term of the same order as the other terms leading to enhanced sensitivity to $\gamma$.

The rates for $f_{D}=K^{-} \pi^{+} \pi^{+} \pi^{-}$are of a similar form, however, an additional coherence parameter, $R_{K 3 \pi}$, is introduced [11] to account for the
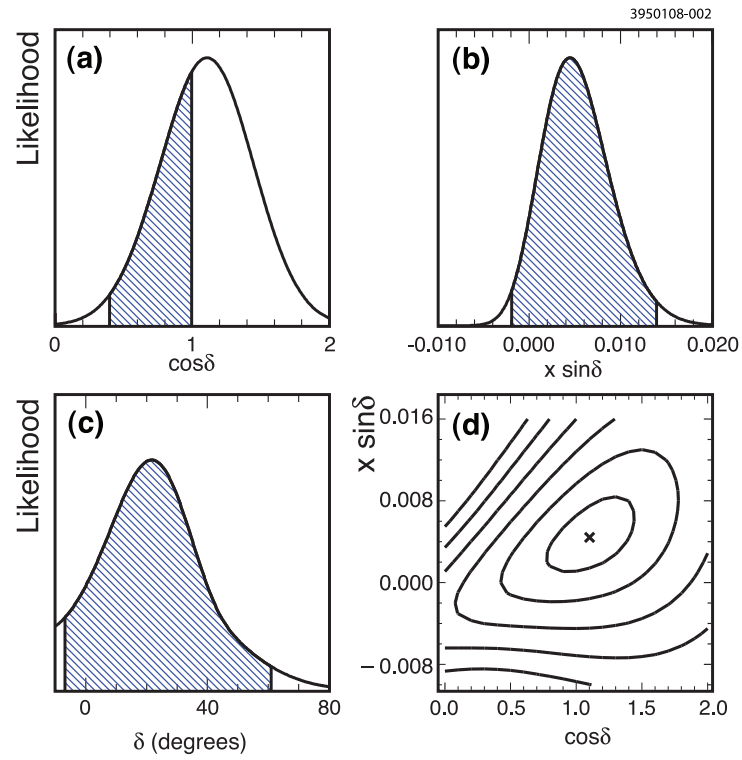

Figure 1. Extended fit likelihood including both statistical and systematic uncertainties for $\cos \delta$ (a), $x \sin \delta(\mathrm{b}), \delta$ (c) and simultaneous likelihood for $\cos \delta$ and $x \sin \delta$ (d) shown as contours in increments of $1 \sigma$. The hatched regions contain $95 \%$ of the area in the physical regions.

possibility of several different intermediate states, with differing strong phases, contributing, such as $K^{* 0} \rho^{0}$ and $K(1270)_{1}^{-} \pi^{+}$. For example the suppressed rate is given by:

$$
\begin{aligned}
& \Gamma_{B^{-} \rightarrow D\left(K^{+} \pi^{-} \pi^{-} \pi^{+}\right) K^{-}} \propto r_{B}^{2}+\left(r_{D}^{K 3 \pi}\right)^{2} \\
& +2 r_{B} r_{D}^{K 3 \pi} R_{K 3 \pi} \cos \left(\delta_{B}+\delta_{D}^{K 3 \pi}-\gamma\right),
\end{aligned}
$$

where $r_{D}^{K 3 \pi}$ is the absolute of the ratio of DCS to $\mathrm{CF}$ amplitudes and $\delta_{D}^{K 3 \pi}$ is the average strongphase difference.

The definition of $R_{K 3 \pi}$ is such that it takes a value between zero and one, with values approaching zero corresponding to many different intermediate states contributing equally and values approaching one corresponding to the dominance of a single intermediate final state. To exploit fully the sensitivity to $\gamma, \delta_{B}$ and $r_{B}$ in the case of $D \rightarrow K^{-} \pi^{+} \pi^{+} \pi^{-}$external measurements of the coherence parameter are extremely 
important.

The quantum correlated $D^{0} \overline{D^{0}}$ data allow direct measurements of $R_{K 3 \pi}$ and $\delta_{D}^{K 3 \pi}$. Doubletagged rates are used to determine these parameters; their dependence on the parameters are listed in Table 4. The interpretation of the measurement of $K^{ \pm} \pi^{\mp} \pi^{-} \pi^{+}$vs. $K^{ \pm} \pi^{-}$requires the meaurement of $\delta$ presented in Section 3 .

The analysis has been performed on $818 \mathrm{pb}^{-1}$ of data and proceeds in a similar manner to that presented in Section 3. All the CP-tags listed in Table 2 are used in this analysis apart from $K_{L}^{0} \pi^{0}$; this and other $K_{L}^{0}$ modes will be included in an updated version of the analysis. In addition, the $C P$-tags $K_{S}^{0} \phi$ and $K_{S}^{0} \eta^{\prime}$ are included. The backgrounds levels are between 1 and $7 \%$ depending on the final state reconstructed. The efficiency for reconstruction for the different final states varies between 4 and $30 \%$.

The different constraints on $R_{K 3 \pi}$ and $\delta_{D}^{K 3 \pi}$ determined from the data are given in Table 4. The combined result for the nine $C P$ tagged rates is shown; the combination is performed including systematic uncertainties and accounting for all correlations amongst the measurements. The combination of the three different constraints leads to the likelihood contours in $R_{K 3 \pi}$ and $\delta_{D}^{K 3 \pi}$ space shown in Figure 2, The most likely values of the parameters are $R_{K 3 \pi}=0.2$ and $\delta_{D}^{K 3 \pi}=144^{\circ}$. A low value of the coherence parameter is favoured. Low coherence dilutes the sensitivity to $\gamma$ of $B \rightarrow D\left(K^{ \pm} \pi^{\mp} \pi^{-} \pi^{+}\right) K$ decays, however, the measurements are very sensitive to $r_{B}$ which is a common parameter with other $B \rightarrow D K$ decays. The usefulness of these constraints on $R_{K 3 \pi}$ and $\delta_{D}^{K 3 \pi}$ can be illustrated when they are combined with the expected yields for $B \rightarrow D(K \pi) K$ and $B \rightarrow D(K 3 \pi) K$ at the LHCb experiment 121314 to determine $\gamma$. Depending on the parameter values the uncertainty on $\gamma$ reduces by 25 to $35 \%$. More details of this analysis are documented in Ref. [15].

\section{Studies of the strong-phase variation in $D \rightarrow K_{S}^{0} \pi^{+} \pi^{-}$}

The process $B^{ \pm} \rightarrow D\left(K_{S}^{0} \pi^{+} \pi^{-}\right) K^{ \pm}$currently provides the best determinations of $\gamma[1617]$. The

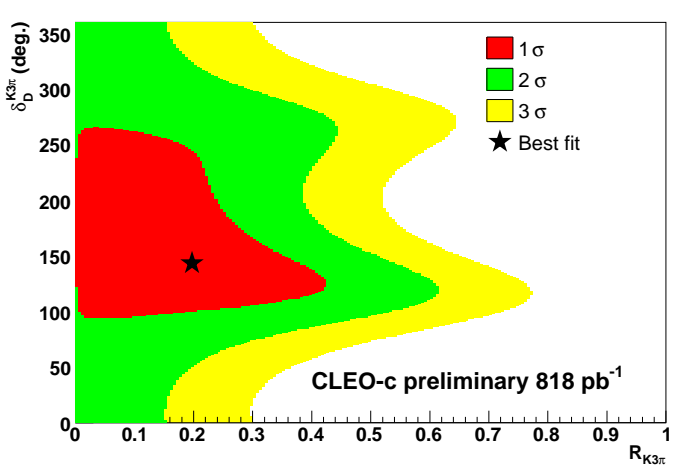

Figure 2. The limits on $R_{K 3 \pi}$ and $\delta_{D}^{K 3 \pi}$ at the 1 , 2 and $3 \sigma$ level.

sensitivity to $\gamma$ is exploited via likelihood fits to the $K_{S}^{0} \pi^{+} \pi^{-}$Dalitz plot [18. These fits require models of the $D^{0} \rightarrow K_{S}^{0} \pi^{+} \pi^{-}$amplitudes which introduce a $5^{\circ}$ to $10^{\circ}$ systematic uncertainty on $\gamma$. Future measurements of $\gamma$ will be limited by this uncertainty.

An alternative method is to perform a modelindependent binned analysis of the Dalitz plots, which was orginally proposed in Ref. 18, and has been developed significantly in Ref. [19. The binned method requires knowledge of two parameters in each bin of the Dalitz space, $c_{i}$ and $s_{i}$, which are the average cosine and sine of the strong-phase difference between $D^{0}$ and $\overline{D^{0}}$ decaying to $K_{S}^{0} \pi^{+} \pi^{-}$, respectively.

The values of $c_{i}$ and $s_{i}$ can be determined from $\psi(3770)$ data at CLEO-c 20]. The value of $c_{i}$ can be measured from differences in the $\mathrm{CP}+$ and $C P-$ tagged Dalitz plots; $s_{i}$ and $c_{i}$ are determined from double $K_{S}^{0} \pi^{+} \pi^{-}$vs. $K_{S}^{0} \pi^{+} \pi^{-}$events.

The measurement of $c_{i}$ and $s_{i}$ from CLEO-c's $818 \mathrm{pb}^{-1}$ sample of $\psi(3770)$ data is underway. Again $M$ and $\Delta E$ are the main variables used to isolate the signal. The $C P$-tagged Dalitz plots and $\pi^{+} \pi^{-}$invariant-mass squared distributions are shown in Figure 3 . The presence of the quantum correlations is shown clearly by the absence of the $\rho^{0}$ in the $C P$ - tagged data.

Preliminary estimates indicate that these data will lead to a 3 to $5^{\circ}$ uncertainty on $\gamma$ from those on the measurements of $c_{i}$ and $s_{i}$ at CLEO-c. The measurements can be improved by including information from $K_{L}^{0} \pi^{+} \pi^{-}$decays at CLEO-c. 
Table 4

Double-tagged rates of interest and their dependence $R_{K 3 \pi}$ and $\delta_{D}^{K 3 \pi}$. The background subtracted yields from the $818 \mathrm{pb}^{1}$ are shown along with the corresponding result for each measurement. Uncertainties are statistical and systematic, respectively.

\begin{tabular}{lcc}
\hline$K^{ \pm} \pi^{\mp} \pi^{+} \pi^{-}$vs. & Measurement & Signal yield \\
\hline$K^{ \pm} \pi^{\mp} \pi^{+} \pi^{-}$ & $R_{K 3 \pi}^{2}=0.00 \pm 0.16 \pm 0.07$ & $30 \pm 6$ \\
$C P$-tags & $R_{K 3 \pi} \cos \delta_{D}^{K 3 \pi}=-0.60 \pm 0.19 \pm 0.24$ & $2183 \pm 47$ \\
$K^{ \pm} \pi^{\mp}$ & $R_{K 3 \pi} \cos \left(\delta-\delta_{D}^{K 3 \pi}\right)=-0.20 \pm 0.23 \pm 0.09$ & $38 \pm 6$ \\
\hline
\end{tabular}
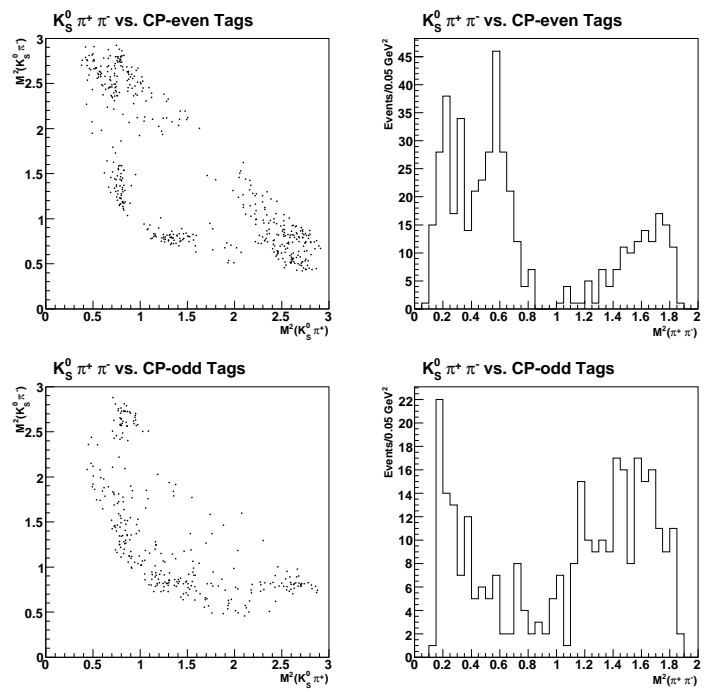

Figure 3. The distributions of the $K_{S}^{0} \pi^{+}$ invariant-mass squared vs. $K_{S}^{0} \pi^{-}$invariant-mass squared (left) and $\pi^{+} \pi^{-}$invariant-mass squared for $C P$-even tagged (upper) and $C P$-odd tagged (lower) $K_{S}^{0} \pi^{+} \pi^{-}$events. The data sample corresponds to a luminosity of $818 \mathrm{pb}^{-1}$.

\section{REFERENCES}

1. J. Charles et al. (CKMfitter Group), Eur. Phys. J. C41, 1 (2005), updated results and plots at http://ckmfitter.in2p3/fr.

2. W.-M. Yao, Journal of Physics G 33, 12006.

3. G. Viehauser et al., Nucl. Instrum. Methods Phys. Res., Sect. A 462, 146 (2001); R. A. Briere et al. (CESR-c and CLEO-c Taskforces, CLEO-c Collaboration), Cornell University, LEPP Report No. CLNS 01/1742 (2001) (unpublished).

4. M. Charles, these proceedings.
5. D. Atwood, I. Dunietz and A. Soni, Phys. Rev. Lett. 783257 (1997); D. Atwood, I. Dunietz and A. Soni, Phys. Rev. D 63 036005 (2001).

6. D. Asner and W. Sun, Phys. Rev. D 73, 034024 (2006); 77, 019901(E) (2008).

7. J. L. Rosner et al., Phys. Rev. Lett. 100, 221801 (2008); D. Asner et al., to be published in Phys. Rev. D, arXiv:0802.2268 [hep$\mathrm{ex}$.

8. Q. He et al., Phys. Rev. Lett. 100, 091801 (2008).

9. T. E. Coan et al., Phys. Rev. Lett. 95, 181802 (2005).

10. A. J. Schwartz, arXiv:0803.0082 [hep-ex].

11. D. Atwood and A. Soni, Phys. Rev. D 68 033003 (2003).

12. LHCb Technical Proposal, LHCb Collaboration, CERN/LHCC 98-4 (1998); LHCb Reoptimized Detector, Design and Performance, LHCb Collaboration, CERN/LHCC 2003-040 (2003).

13. M. Patel, LHCb-2006-066.

14. A. Powell, LHCb-2007-004.

15. A. Powell, arXiv:0805.1722 [hep-ex].

16. B. Aubert et al., arXiv:0804.2089 [hep-ex].

17. K. Abe et al., arXiv:0803.3375 [hep-ex].

18. A. Giri, Yu. Grossman, A. Soffer and J. Zupan, Phys. Rev. D 68054018 (2003).

19. A. Bondar and A. Poluektov, arXiv:0801.0840 [hep-ex]; A. Bondar and A. Poluektov, Eur. Phys. J. C47 347 (2006).

20. E. White and Q. He, arXiv:0711.2285v1 [hepex]. 